\title{
Pubertal Testosterone and Brain Response to Faces in Young Adulthood: An Interplay between Organizational and Activational Effects in Young Men
}

\author{
Zhijie Liao, ${ }^{1,2}$ Steven Tilley, ${ }^{2}$ Andrei Mouraviev, ${ }^{2}$ Ammar Khairullah, ${ }^{3}$ and Tomas Paus ${ }^{1,2,3}$ \\ ${ }^{1}$ Department of Psychology, University of Toronto, Toronto, Ontario M5S 3G3, Canada, ${ }^{2}$ Bloorview Research Institute, Holland Bloorview Kids \\ Rehabilitation Hospital, Toronto, Ontario M4G 1R8, Canada, and ${ }^{3}$ Department of Psychiatry, University of Toronto, Toronto, Ontario M5T 1R8, Canada
}

According to the organizational-activational hypothesis, the organizational effects of testosterone during (prenatal) brain development moderate the activational effects of adult testosterone on behavior. Accumulating evidence supports the notion that adolescence is another period during which sex hormones organize the nervous system. Here we investigate how pubertal sex hormones moderate the activational effects of adult sex hormones on social cognition in humans. To do so, we recruited a sample of young men $(n=507$; age, $\sim 19$ years) from a longitudinal birth cohort and investigated whether testosterone exposure during adolescence (from 9 to 17 years of age) moderates the relation between current testosterone and brain response to faces in young adulthood, as assessed with functional magnetic resonance imaging (fMRI). Our results showed that the cumulative exposure to testosterone during adolescence moderated the relation between adult testosterone and both the mean fMRI response and functional connectivity (i.e., node strength). Specifically, in participants with low exposure to testosterone during puberty, we observed a positive relationship between current testosterone and the brain response to faces; this was not the case for participants with medium and high pubertal testosterone. Furthermore, we observed a stronger relationship between the brain response and current testosterone in parts of the angry-face network associated with (vs without) motion in the eye region of an observed (angry) face. We speculate that pubertal testosterone modulates the relationship between current testosterone and brain response to social cues carried by the eyes and signaling a potential threat.

Key words: ALSPAC; facial expression; fMRI; puberty; testosterone

\section{Significance Statement}

Accumulating evidence supports the organizational effects of pubertal testosterone, but the body of literature examining these effects on social cognition in humans is in its infancy. With a sample of young men from a longitudinal birth cohort, we showed that the cumulative exposure to testosterone during adolescence moderated the relation between adult testosterone and both the mean BOLD signal change and functional connectivity. Specifically, we observed a positive relationship between adult testosterone and the brain response to faces in participants with low exposure to testosterone during puberty, but not in participants with medium and high pubertal testosterone. Results of further analysis suggest that sensitivity to cues carried by the eyes might underlie the relationship between testosterone and brain response to faces, especially in the context of a potential threat.

Received Jan. 23, 2020; revised Jan. 18, 2021; accepted Jan. 25, 2021.

Author contributions: T.P. designed research; T.P. performed research; Z.L., S.T., A.M., and A.K. analyzed data; S.T., A.K., and T.P. edited the paper; Z.L. wrote the paper.

The UK Medical Research Council and Wellcome (Grant 102215/2/13/2) and the University of Bristol provide core support for the Avon Longitudinal Study of Parents and Children (ALSPAC). A comprehensive list of grant funding is available on the ALSPAC website (http://www.bristol.ac.uk/alspac/external/documents/grant-acknowledgements.pdf). This research was specifically funded by the Wellcome Trust and the Medical Research Council (Grant 076467/Z/05/Z). The IMAGEN consortium contributed to collecting data of IMAGEN, and its membership can be found in https:// imagen-europe.com/resources/author-list/. This research was supported by a grant from the National Institutes of Health (Grant R01-MH-085772 to T.P.). Z.L. is supported by China Scholarship Council Award 201806380177. This article does not necessarily represent the official views of the National Institutes of Health. We thank all of the families who took part in this study and the whole ALSPAC team, which includes midwives, interviewers, computer and laboratory technicians, clerical workers, research scientists, volunteers, managers, receptionists, and nurses. We also thank Jean Shin for providing very useful suggestions on statistical analysis.

The authors declare no competing financial interests.

Correspondence should be addressed to Tomas Paus at tpausresearch@gmail.com.

https://doi.org/10.1523/JNEUROSCI.0190-20.2021

Copyright $(2021$ the authors

\section{Introduction}

Testosterone is known to influence the regulation of emotional responses and to mediate some of the sex differences in social cognition and behavior (Eisenegger et al., 2011). The action of testosterone in the brain is both organizational and activational. According to the original organizational-activational hypothesis and subsequent studies, organizational effects refer to the ability of sex hormones to sculpt the brain during the perinatal period in a manner that persists into the adulthood; activational effects refer to transient effects of the sex hormones on facilitating behavior in specific social contexts in later life (Phoenix et al., 1959; Wallen, 2009). But research over the past decades has revealed that, in addition to the perinatal period, sex hormones may organize the nervous system also during adolescence (Sisk 
and Zehr, 2005; Schulz et al., 2009a; Herting et al., 2014; Schulz and Sisk, 2016). Compared with male rodents with high levels of pubertal testosterone, those who experienced low exposure to testosterone during puberty not only displayed fewer masculine behaviors (Shrenker et al., 1985; Eichmann and Holst, 1998; Schulz et al., 2009b), but also showed a diminished response to testosterone treatment in adulthood with regard to aggressive and/or sexual behaviors (Schulz et al., 2009b). Furthermore, the reduction in masculine behaviors could not be reversed by a prolonged period of testosterone replacement in adulthood (Schulz et al., 2004). These animal studies support the idea that the organizational effects of testosterone during puberty facilitate its action on social behavior and cognition later in life. By experiencing changes in internal (e.g., hormonal level) and external (e.g., social relationships) environments, it has been argued that adolescence is characterized by heightened emotional reactivity and sensitivity to social interactions (Guyer et al., 2016). A growing body of studies in humans has provided evidence of testosterone-related variations in brain function during adolescence (for review, see Vijayakumar et al., 2018). For example, pubertal testosterone is associated with changes in brain response to facial expression (Vijayakumar et al., 2019), and with threat-related amygdala-orbitofrontal cortex coupling in adolescence (Spielberg et al., 2015). No human studies have investigated, however, the interactions between the pubertal and adult testosterone vis-à-vis social cognition or behavior.

Faces are the most apparent social cues in daily life and provide information about the mental and emotional states of people (Mandal and Awasthi, 2015). Extracting and processing information from a face require the coordinated engagement of many brain regions (Haxby et al., 2000; Adolphs, 2002; Tahmasebi et al., 2012). Eyes, as "windows to the soul," play an important role in transmitting social cues on faces (Hietanen, 2018). An inability to make use of information from the eye region of a face can lead to failures in recognizing facial expression and face identity (Adolphs, 2002; Itier and Batty, 2009). Studies also showed a positive correlation between the time spent fixating on the eye region of faces and the brain response to faces in several regions, such as the fusiform gyrus and amygdala (George et al., 2001; Dalton et al., 2005). Amygdala responds strongly when directing an observer's gaze toward (vs away from) the eyes of a fearful face (Gamer and Büchel, 2009).

Relationships between testosterone levels and brain response to facial expression have been observed in both adolescents (see above) and adults. The latter findings are inconsistent, however, showing both positive (Derntl et al., 2009; Manuck et al., 2010) and negative (Stanton et al., 2009; Buades-Rotger et al., 2016) relationships between testosterone levels and the amygdala response to faces. In addition to the small sample sizes of these studies, an unaccounted organizational effect of testosterone might have also contributed to such inconsistencies.

In this study, we recruited a sample of young men $(n=507)$ from a longitudinal birth cohort and investigated whether pubertal testosterone moderates the relation between current testosterone and brain response to faces in early adulthood (age, $\sim 19$ years), as assessed with functional magnetic resonance imaging (fMRI).

\section{Materials and Methods}

Participants. The sample of young men studied here was drawn from the Avon Longitudinal Study of Parents and Children (ALSPAC). This is a birth cohort that recruited pregnant women resident in Avon county, United Kingdom, with an expected delivery date between April 1, 1991, and December 31, 1992. Since then, the children and their parents have been studied extensively, and longitudinal data including self-administered questionnaires and clinical examinations collected during study visits are available (Boyd et al., 2013; Fraser et al., 2013). Blood samples were taken at select visits over the course of the study, approximately at the following ages: 7, 9, 11, 13, 15, and 17 years. Ethical approval for the study was obtained from the ALSPAC Law and Ethics Committee and the Local Research Ethics Committees. Please note that the study website contains details of all the data that are available through a fully searchable data dictionary and variable search tool (http://www.bristol.ac.uk/alspac/researchers/our-data/).

As part of the ALSPAC project, we recruited 507 male participants, who were $\geq 18$ years of age (mean age, $19.62 \pm 0.84$ years) and had at least three blood samples available for the assessment of testosterone levels just before and during their adolescence (any combination of samples obtained at $9,11,13,15$, and 17 years of age). Details of the sampling method have been described in our previous reports (Khairullah et al., 2014; Jensen et al., 2018). After performing quality control on fMRI images and testosterone data, 389 of the initial 507 participants with good quality fMRI $(n=459)$, pubertal $(n=440)$, and current testosterone $(n=495)$ data were included in this analysis. Details of quality control are described below.

Testosterone. To obtain measurements of the average level of bioavailable testosterone across puberty, we assayed the levels of sex steroids in plasma, and estimated a testosterone trajectory from 9 to 17 years for each participant using the shape-preserving spline-interpolation function. The effects of circadian rhythm on testosterone levels were carefully controlled for each visit before estimating the testosterone trajectory (Khairullah et al., 2014). We then calculated a measure of the average level of testosterone by dividing the area under the curve of the testosterone trajectory by the number of months between the first and last blood samples. To ensure robust estimates, this calculation was restricted to participants who had blood samples collected at a minimum of four visits $(n=440)$ and excluded participants with only three blood samples. Note that, in participants with four samples, values of average level of testosterone varied as a function of the visit with a missing sample. To correct for this bias, we included the "missing visit" as a dummy variable in the model of the average level of testosterone. In subsequent analyses, we used the corrected values (mean + residual) in this "four-samples" subgroup. Furthermore, we observed no differences in the average level of testosterone between subgroups of participants with five and four samples, respectively $(t=1.144, \mathrm{df}=410.53, p=0.253)$. To derive values of bioavailable testosterone, we used the equation from the study by Södergård et al. (1982) in conjunction with the sex hormone-binding globulin concentrations and adjusted values of total testosterone (Khairullah et al., 2014). In the text below, we refer to this measure of bioavailable testosterone as "pubertal T."

At the time of MRI scan, participants were asked to give a sample of saliva to measure their current testosterone level: "current T." Each participant provided one cryovial of saliva (Salimetrics) collected via passive drool; participants were instructed not to eat, drink, smoke, or chew gum for $30 \mathrm{~min}$ before giving the saliva sample. Saliva samples were placed immediately in the freezer and were sent to the Biomarker Core Laboratory at The Pennsylvania State University, where ELISAs were conducted using commercially available kits to determine salivary concentrations of testosterone (Testosterone Enzyme Immunoassay Kit \#12402 , Salimetrics). The assay range is $6.1-600 \mathrm{pg} / \mathrm{ml}$ with sensitivity of $1 \mathrm{pg} / \mathrm{ml}$, with average interassay and intra-assay coefficients of variation of $<10 \%$. To convert current $\mathrm{T}$ (in picograms per milliliter) to SI unit (in nanomol per liter), we multiplied the value by 0.00347 . Among the 507 participants, 12 were excluded because of missing samples, with levels below detection capability of assay or being outliers.

MRI acquisition and preprocess. Scanning was performed on a $3 \mathrm{~T}$ magnet HDx System (General Electric). High-resolution T1-weighted (T1w) anatomic images were acquired using 3D FSPGR (fast spoiled gradient-echo) sequences (resolution $=1 \times 1 \times 1 \mathrm{~mm}$; slice thickness $=1$ $\mathrm{mm} ; \mathrm{TR}=7.9 \mathrm{~ms}$ ). Functional T2 $*$-weighted images were acquired using gradient echo (GE)-echoplanar imaging $(\mathrm{EPI})$ sequences (resolution = $3.4 \times 3.4 \mathrm{~mm}$; slice thickness $=2.4 \mathrm{~mm}$; TR $=3000 \mathrm{~ms}$ ). During the $\mathrm{fMRI}$ session, participants passively viewed short video clips displaying ambiguous facial expressions (with face movements such as nose twitching, 
opening mouth, blinking eyes), angry facial expression or nonbiological control stimuli (consisting of black and white concentric circles of various contrasts, expanding and contracting at various speeds; Grosbras and Paus, 2006). The three viewing conditions were organized into 19 blocks of $18 \mathrm{~s}$ duration each ( 5 ambiguous, 5 angry, and 9 control; each face block contains seven to eight video clips) for a total of 130 volumes in a single 6 min fMRI run.

Of 507 participants, a total of 496 completed both the face paradigm and T1-weighted scan. Quality control and preprocessing of anatomic and functional data were performed, respectively, using MRIQC 0.15.0 (Esteban et al., 2017), FMRIPrep 1.3.2 (Esteban et al., 2019), and FSL_regfilt 5.0.9. A total of 29 participants were excluded because of excessive head movement during functional MR scanning. Excessive head movement was defined as meeting one or more of the following criteria: mean framewise displacement (FD) was 2 SDs above average; the number of volumes with FD $>0.5 \mathrm{~mm}$ was 2 SDs above average; and the number of volumes with $\mathrm{FD}>5 \mathrm{~mm}>0$. In addition, eight participants were excluded because of poor quality of functional image and failure in registration. Thus, the final fMRI dataset consists of 459 participants.

After initial quality control, FMRIPrep was used to produce a nonlinear mapping from the ICBM 152 Nonlinear Asymmetrical template (version 2009c) to the T1w space for each participant. These mappings were used to transform the masks of regions of interest (ROIs; see below) to each participant's T1w space. As the ROIs were originally defined in the linear ICBM 152 template, an affine transformation from the linear to the nonlinear templates was applied as part of the transformation step. Advance normalization tools were used for the affine registration and the transformations. Functional images were corrected for slice time, susceptibility distortions, and head motion. This correction was followed by coregistration to the corresponding T1w using boundarybased registration with $9 \mathrm{df}$. Automatic removal of motion artifacts using independent component analysis (ICA-AROMA; Pruim et al., 2015) was performed, and corresponding noise regressors were collected that were used to perform "nonaggressively" denoised runs on preprocessed functional images in T1w space with FSL_regfilt. For further details of the pipeline, including the software packages used by FMRIPrep for each preprocessing step, please refer to the online documentation (https:// fmriprep.readthedocs.io/en/stable/). All analyses reported here used functional data extracted from the participant's native T1w space.

MRI analysis with face-related regions of interest. All subsequent analyses were limited to a set of 25 ROIs defined by a probabilistic map computed from data obtained with the same paradigm in a large sample of adolescents $(n=1110)$ participating in the IMAGEN Study (Tahmasebi et al., 2012). In this probabilistic map, 21 ROIs were identified by their consistent (population probability, $>0.5$ ) engagement during the ambiguous and angry face processing, relative to the control condition. For completeness, the present analyses also include four contralateral homologs (population probability, $<0.5$ ) of unilateral ROIs featuring in this probability map; hence, a total of 25 ROIs. The ROIs included the following brain regions in both the left hemisphere (L) and right hemisphere (R; Extended Data Fig. 3-1): amygdala (AmygdalaL, AmygdalaR), cerebellum (CerebellumL, CerebellumR), putamen (PutamenL, PutamenR), rhinal sulcus (RhinalSulcusL, RhinalSulcusR), anterior superior temporal sulcus (AntSTSL, AntSTSR), posterior temporal sulcus (PostSTSL, PostSTSR), fusiform face area (FFAL, FFAR), lateral occipital cortex (LOCL, LOCR), V2-V3 (V2V3L, V2V3R), premotor cortex (PMCL, PMCR), midventrolateral frontal cortex (MVLFCL, MVLFCR), mid-dorsolateral frontal cortex (MDLFCL, MDLFCR), and presupplementary motor area (PreSMAR). The probabilistic maps computed previously (Tahmasebi et al., 2012) were used to extract the mean percentage of BOLD (blood oxygenation level-dependent) signal change and mean BOLD signal time series within each of the 25 ROIs. The values in the probabilistic maps indicate the probability of a voxel showing a BOLD response to faces in a large $(n>1000)$ sample of adolescents.

The denoised preprocessed functional images were entered into the individual (fixed-effects) general linear model analysis performed with FSL 6.0.1. The following two contrasts were considered in this study: (1) angry faces versus control stimuli (Angry vs Control); and (2) ambiguous faces versus control stimuli (Ambiguous vs Control). Double- gamma was selected as the hemodynamic response function. For each ROI, the mean percentage BOLD signal change (\%BSC) within the ROI was extracted for all participants, as in the study by Tahmasebi et al. (2012).

To investigate whether testosterone levels are associated with the magnitude of the contribution of each region to the "face network," we extracted mean BOLD signal time series from each of 25 ROIs and used these to calculate matrixes of "functional connectivity." Specifically, for each ROI, the mean BOLD signal time series was obtained by weighted mean (weighted by probabilities) of the BOLD signal from all voxels constituting the ROI at every time point (130 time points in total). Subsequently, we created the BOLD signal time series for each face condition by concatenating the mean-centered signal from the corresponding blocks, shifted by 2 TRs $(6 \mathrm{~s})$ to accommodate for the rise in the hemodynamic response. Then we generated a correlation matrix based on the time series from the 25 ROIs for each participant; all correlation coefficients in the matrix were Fisher $z$ transformed. This step yielded a $25 \times 25$ symmetric functional connectivity matrix for each participant and each of the two face conditions. We reduced these matrices into an undirected weighted "face network" by zeroing out negative correlations. Finally, we calculated node strength (i.e., sum of all edges) for each ROI (node) to summarize the graph for each participant. This calculation was conducted using R software (version 3.5.2) with the "igraph" package (Pemberton, 1975).

Brain response to faces associated with motion in the eye region in an observed face. As we found that there was a relationship between the brain response to faces and testosterone in the low pubertal-T group, we hypothesized that brain response in parts of the face network associated with motion in the eye region in an observed face may underlie this relationship; such motion can act as a carrier of additional social cues (Adolphs, 2002; Gamer and Büchel, 2009). We tested this hypothesis by comparing the strength of the correlation between brain response to faces and current testosterone in parts (voxels) of the face network regions associated with processing motion in the eye region in the observed faces, compared with parts (voxels) of the face network not associated with such motion in eye region in observed faces. To identify these "eyes" and "non-eyes" subregions (voxels) of the face network, we used an independent dataset (IMAGEN; see below) to create probabilistic maps of the brain response to faces when the observed face exhibited motion in the eye region (Fig. 1, workflow). Using the same face paradigm, we analyzed fMRI time series in an event-related manner with motion in the eye region (in the observed face) as an event of interest. To identify segments of the video clips containing motion in the eye region, we asked five raters (three of the five being males) to identify motion in the eye region in faces in the 10 video clips ( 5 angry video clips with a total of 37 faces, 10 ambiguous video clips with a total of 36 faces) by watching the video clips frame by frame. There were 541 frames with a $33 \mathrm{~ms}$ frame interval for each video clip. To match the visual condition of fMRI scanning, raters were asked to view the video clips with the height of the face of $\sim 10 \mathrm{~cm}$ and the distance between their eyes and a computer screen of $\sim 60 \mathrm{~cm}$; thus, each face subtending a visual angle of $\sim 10^{\circ} \times 7^{\circ}$. Frames with motion in the eye region (i.e., a change in position compared with the previous frame) were marked as " 1 " for a motion and " 0 " for no motion. Subsequently, the time series of the motion rating from each rater were smoothed by convolution operation with a Gaussian kernel: two frames before and after a "motion" (i.e., $1)$ frames were also coded as 1 . Fleiss' kappa tests indicated moderate to substantial agreement among the five raters $(\kappa$ values are $0.51 \sim 0.79$ with an average of $0.65 ; \mathrm{McHugh}, 2012$ ). To generate input functions for the event-related design, we averaged ratings from the five raters and binarized it with a threshold of $>0.5$. These time series of binary ratings (Extended Data Fig. 1-1) for each video clip were used to derive angryeye, ambiguous-eye, no-motion event inputs that were, together with the nonbiological control events, applied to an event-related fMRI analysis to derive maps of brain response to motion in the eye region in the observed face (angry and ambiguous).

To generate the above probabilistic maps, we used data acquired during the first wave of the IMAGEN Study, which included a total of 2080 typically developing adolescents, 13-15 years of age (Schumann et al., 


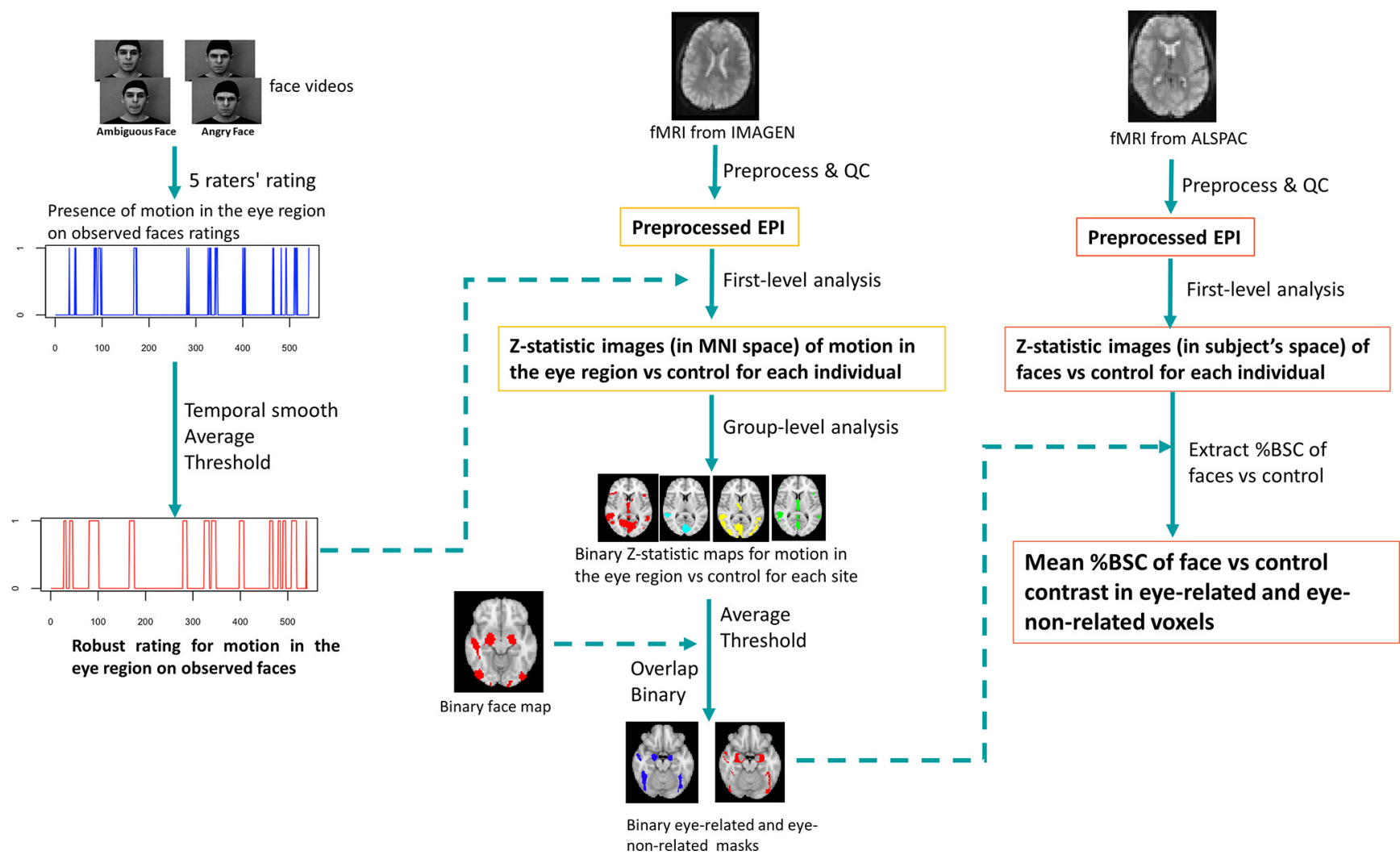

Figure 1. Workflow of assessing correlation between \%BSC and testosterone in parts of the face network associated (or not) with motion of the eyes in the observed face. We identified motion in the eye region in faces in the face video clips frame by frame (left; Extended Data Figure 1-1, details). The rating of motion was used to obtain a map of the brain response to motion in the eye region in the observed face using whole-brain group analysis with IMAGEN data (middle; Extended Data Figure 1-2, information of the IMAGEN dataset). With this "motion in the eye region" map, all voxels constituting the face network ( 25 ROls, as described above) were classified as overlapping (or not) with the motion in the eye region map, thus generating the following two sets of voxels for each (ambiguous and angry) face network: (1) voxels associated with motion in the eye region in the observed face; and (2) voxels not associated with motion in the eyes in the observed face (middle bottom). Subsequently, these eye-related and eye-nonrelated voxels were used to extract mean \%BSC in ALSPAC data (right).

2010). Participants and their families were recruited at eight sites located in England (two sites), France (one site), Ireland (one site), and Germany (four sites). Scanning was performed on $3 \mathrm{~T}$ scanners from four different manufacturers (Siemens, four sites; Philips, two sites; General Electric, one site; Bruker, one site). High-resolution T1weighted anatomic images were acquired using 3D MPRAGE (magnetization prepared rapid acquisition gradient echo) sequence $(\mathrm{TR}=$ $2300 \mathrm{~ms}$; TE $=2.8 \mathrm{~ms}$ ). Functional images were acquired using GE-EPI sequences (slice thickness, $2.4 \mathrm{~mm}$; slice gap, $1.0 \mathrm{~mm}$; TE = $30 \mathrm{~ms}$; TR = $2200 \mathrm{~ms}$ ), and with the same face-task paradigm as in ALSPAC (Tahmasebi et al., 2012). Since only males are included in the ALSPAC dataset, we conducted this analysis only in males as well. For the current report, structural and functional MRI data were available in 1021 males; MRI data obtained in 871 adolescents were retained after excluding 150 participants who failed quality control of the preprocessed images (Extended Data Fig. 1-2, distribution of the qualified participants across the eight acquisition sites).

Preprocessing procedures that were applied to the IMAGEN dataset were similar to those for the ALSPAC dataset. In short, functional images were corrected for slice time, susceptibility distortions, head motion, and noise regressors detected by ICA-AROMA. The motioncorrected and denoised EPI data were spatially smoothed with an isotropic Gaussian kernel of $6 \mathrm{~mm}$ full-width at half-maximum using FMRI expert analysis tool (FEAT). With FLIRT (FMRIB Linear Image Registration Tool) inside FEAT, the preprocessed images from EPI and the high-resolution T1-weighted images of each participant were aligned together using $12 \mathrm{df}$ linear registration. The high-resolution anatomic images were aligned, with nonlinear registration, to the MNI-152 standard space template. Then EPI volumes were entered into the first-level analysis (i.e., individual general linear model). For each individual, we computed $z$-statistic maps for the following two contrasts: (1) angry-eye versus control; and (2) ambiguous-eye versus control. The $z$-statistic maps indicated how much stronger the brain response to motion in the eye region in the observed face was compared with nonbiological stimulus. Subsequently, the two $z$-statistic maps in standard space for each individual were submitted to group-level analysis with FLAME (FMRIB Local Analysis of Mixed Effects; in FEAT) to generate probabilistic maps of the brain response to motion in the eye region in the observed face. Individuals from the same acquisition site were submitted to the same group analysis, for a total of eight group analyses. The resulting $z$-statistic images were thresholded by $z>3.1$ and cluster $p$ value $>0.05$, binarized, averaged across acquisition sites, and thresholded by $>0.5$. The resulting maps contain brain regions with a high $(>0.5)$ probability of brain response associated with motion in the eye region in the observed angry and ambiguous faces, respectively; in the text below, we refer to these as "angry-eyes" and "ambiguous-eyes" maps.

The angry-eyes and ambiguous-eyes maps were intersected with the binary maps of the brain response to angry and ambiguous faces, respectively (Tahmasebi et al., 2012), to identify voxels of these face maps that were associated (or not) with motion in the eye region in the observed face. Next, we extracted mean \%BSC of Angry versus Control and Ambiguous versus Control contrast from these two sets of voxels; in the text below, we refer to these as "eye-related" and "eye-nonrelated" sets of voxels in each of the two contrasts.

Statistical analysis. In our initial analysis, after correcting for age of participants at scanning and for the time of day of collecting saliva samples, we tested whether there was an interaction between pubertal $\mathrm{T}$ and current $\mathrm{T}$ vis-à-vis face processing; to do so, we used linear regression models [\%BSC (or strength) $\sim$ pubertal $\mathrm{T}+$ current $\mathrm{T}+$ pubertal $\mathrm{T} \times$ current T]. Using this model, we estimated the $\beta$ of the interaction term (i.e., pubertal $\mathrm{T} \times$ current $\mathrm{T}$ ) for each ROI. Before running the model with interaction terms, all variables were standardized so that the $\beta$ 
A Pubertal testosterone in 3 groups

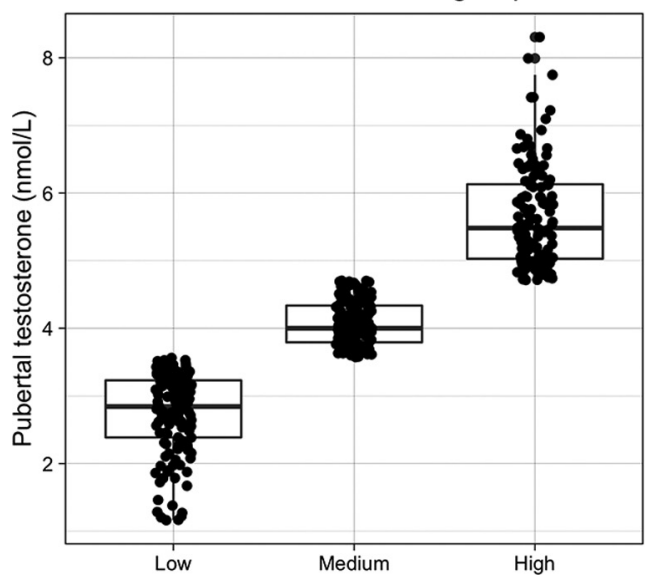

B Current testosterone in 3 groups

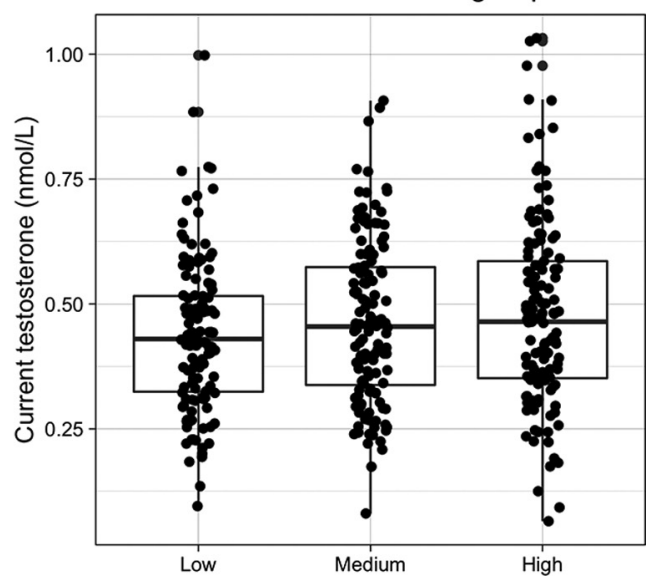

Figure 2. $\boldsymbol{A}, \boldsymbol{B}$, Testosterone levels in the three pubertal T groups: pubertal testosterone $(\boldsymbol{A})$; current testosterone $(\boldsymbol{B})$.

values of interaction terms are comparable. Subsequently, as we are interested in the relationship between testosterone and brain response to faces as a whole rather than region by region, we tested the significance of the interaction $(\beta)$ profiles across the 25 ROIs for each task contrast. This was performed using a permutation approach (Buzkova, 2016) that includes the following steps: (1) fitted the null model without interaction [\%BSC (or strength) pubertal $\mathrm{T}+$ current $\mathrm{T}$ ) and obtained residuals for all 25 ROIs; (2) permuted pubertal T; (3) fitted the interaction model with the permuted pubertal $\mathrm{T}$ and obtain $\beta$ coefficient for the interaction term for each of the 25 ROIs; (4) repeated steps 2 and 35000 times, and then averaged the 5000 simulated $\beta$ values for each of the 25 ROIs to derive a null profile; and (5) used paired $t$ test, as the profiles consist of the same 25 ROIs, to test whether the observed $\beta$ profile was different from the null profile, and thus, obtained the significance level.

We observed that all $\beta$ profiles of the interaction term (pubertal $\mathrm{T} \times$ current $\mathrm{T}$ ) were significant. To investigate details in the directions of moderation effects of pubertal $\mathrm{T}$ on the relation between current $\mathrm{T}$ and brain response to faces, we divided participants into the following three groups based on the tertiles of their pubertal $\mathrm{T}$ level: low pubertal $\mathrm{T}$ (Low, pubertal $\mathrm{T} \leq 33 \mathrm{rd}$ percentile); medium pubertal $\mathrm{T}$ (Medium, 33rd percentile $<$ pubertal $\mathrm{T}<67$ th percentile); and high pubertal T (High, pubertal $\mathrm{T} \geq 67$ th percentile). First, we tested the group difference in current $T$ using ANOVA. Since diurnal and age variation of testosterone levels in men is well documented (Brambilla et al., 2009), we controlled for the age of the participants and the time of collecting saliva samples in the following analysis. Partial correlation coefficients between the \%BSC of the two face contrasts (angry, ambiguous) and current $\mathrm{T}$, with age and time of data collection controlled for, were calculated for each of the 25 ROIs for each group. We then applied a permutation approach to test whether the observed profiles of correlation coefficients across the 25 ROIs were different from a null profile. Specifically, we (1) permuted current $\mathrm{T}$ and then (2) calculated correlation coefficients between the permuted current $\mathrm{T}$ and \%BSC (and strength) to obtain a simulated correlation coefficient profile; (3) repeated steps 1 and 25000 times, and then averaged the 5000 simulated profiles (i.e., averaged the 5000 simulated correlation coefficients for each of the 25 ROIs) to derive a null profile; (4) finally, we used paired $t$ test (25 ROIs) to test whether the observed correlation coefficient profile is different from the null profile, and thus, obtained the significance level [with correction for the false discovery rate (FDR) for three groups] of the relationship between current $\mathrm{T}$ and \%BSC (and strength) across the 25 ROIs. Since we compared the correlation coefficient profiles that consist of the same 25 ROIs, we used repeated-measures ANOVA and post hoc (paired $t$ test) comparisons with the correction of Holm (1979) for the $p$ value to compare group differences in the Fisher $z$-transformed partial correlation coefficients. To replicate the previously reported relation between testosterone and amygdala response to facial expression (Derntl et al., 2009; Stanton et al., 2009), we also ran a separate regression analysis on \%BSC of the two contrasts in bilateral amygdala (2 of the 25 ROIs) and testosterone. The same analyses (calculated partial correlation coefficient, repeatedmeasures ANOVA, and multiple comparisons) were also conducted on the association between the node strength of each of the 25 ROIs and current $\mathrm{T}$, as a function of puberty $\mathrm{T}$ (i.e., tertile-based groups).

We used linear regression model (with age and time of data collection controlled) to test the relationship between current $\mathrm{T}$ and \%BSC in eye-related and eye-nonrelated sets of voxels. The false discovery rate for the three groups was used to correct for multiple comparisons. As we found that there was a positive correlation between current $\mathrm{T}$ and \%BSC of Angry versus Control contrast in eye-related, but not in eye-nonrelated, sets of voxels in the Low group (see below), a further bootstrapping test $(n=5000)$ was applied to verify the difference on the slope of the two correlations. For each bootstrapping replication on current $\mathrm{T}$ and \%BSC within the Low group, we estimated the slopes of current $\mathrm{T}$ and \%BSC in eye-related and eye-nonrelated sets of voxels, respectively. Then a $z$-value was calculated with following formula: mean differences of the two slopes were divided by the SD of the differences of the two slopes. The significance level of the difference on slopes was obtained by applying a one-side test to the $z$-value.

All statistical analyses were conducted using $\mathrm{R}$ software. Values beyond the 1.5 interquartile range from the lower or upper quartile in \% BSC or strength were omitted. The significance threshold for the $p$ value is 0.05 (two sided).

\section{Results}

\section{Summary of testosterone levels}

Participants' mean \pm SD testosterone concentrations at the time of MRI scanning (i.e., current T) were $0.45 \pm 0.17 \mathrm{nmol} / \mathrm{L}$. Average levels of pubertal testosterone (i.e., pubertal $\mathrm{T}$ ) were $4.13 \pm 1.32 \mathrm{nmol} / \mathrm{L}$; Figure $2 \mathrm{~A}$ shows levels of pubertal $\mathrm{T}$ in the three tertile-based groups (Low, Medium, High). Current $\mathrm{T}$ in the Low, Medium, and High groups were, respectively, $0.44 \pm$ $0.15,0.46 \pm 0.16$, and $0.48 \pm 0.19 \mathrm{nmol} / \mathrm{L}$ (mean \pm SD; Fig. $2 B$ ). The ANOVA result showed no differences in current $\mathrm{T}$ across the three pubertal T groups $\left(F_{(2,386)}=2.1, p=0.122\right)$.

\section{Pubertal T modulated the correlation between current $\mathrm{T}$ and \%BSC across all 25 ROIs}

The profiles of interaction term (i.e., pubertal $\mathrm{T} \times$ current $\mathrm{T}$ ) were different from the null profile for both the Angry and Ambiguous contrast (Angry, $p<0.001$; Ambiguous, $p=0.006$ ). In the Angry versus Control contrast, the mean partial correlation coefficients between current $\mathrm{T}$ and \%BSC in the Low, Medium, and High groups were, respectively, $0.110 \pm 0.083$, 

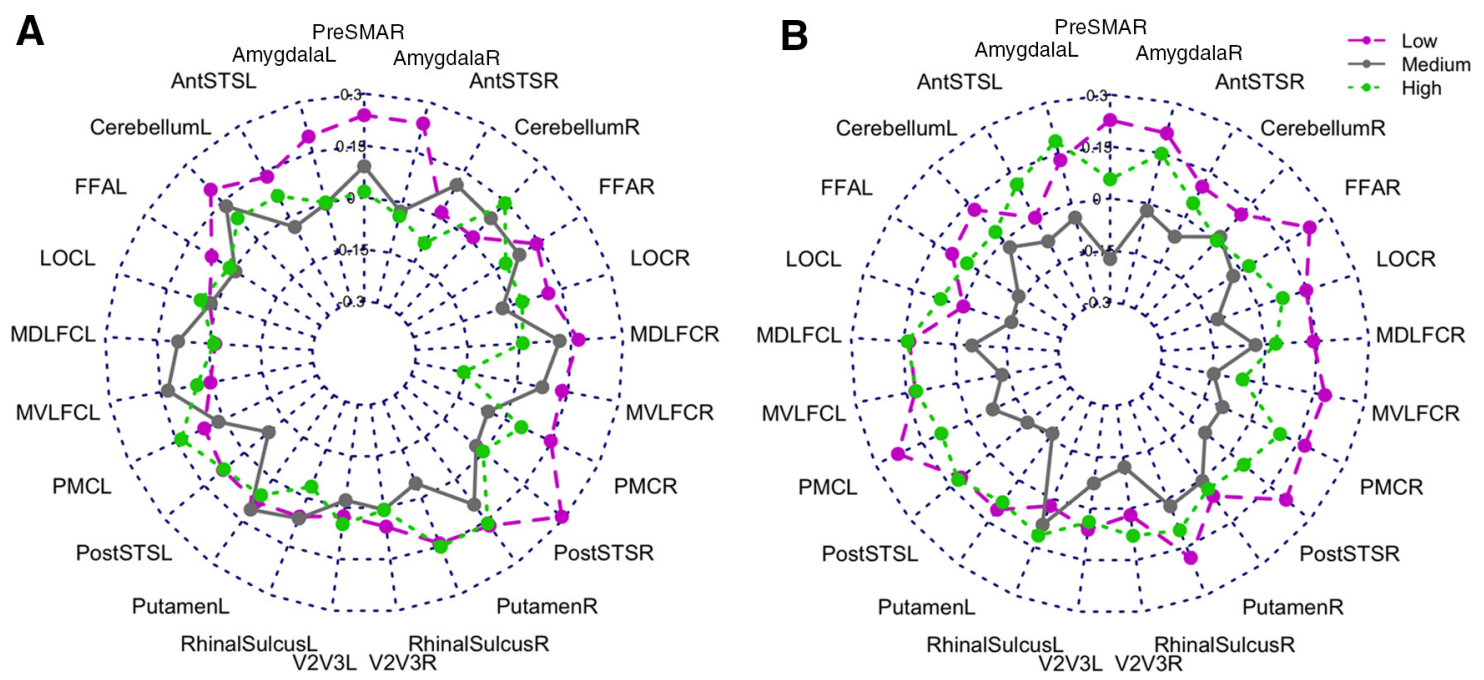

Figure 3. $\boldsymbol{A}, \boldsymbol{B}$, Partial correlation coefficients between \%BSC and current $\mathrm{T}$ for the three pubertal T groups: Angry versus Control contrast $(\boldsymbol{A})$; Ambiguous versus Control contrast ( $\boldsymbol{B})$. Magenta dashed line represents the Low group; gray solid line represents the Medium group; green dotted line represents the High group. Detailed information of the 25 ROls can be found in Extended Data Figure 3-1. Correlation coefficients for each region can be found in Extended Data Figure 3-2.

$0.034 \pm 0.067,0.033 \pm 0.073$ (mean $\pm \mathrm{SD}$ ). The profiles of correlations between \%BSC and current T differed from the null profile in all three groups (Low, $p<0.001$; Medium, $p=0.029$; High, $p=0.038$; FDR corrected; Fig. 3A, Extended Data Fig. $3-2)$. In the Ambiguous versus Control contrast, the mean partial correlation coefficients between current $\mathrm{T}$ and \%BSC in Low, Medium, and High groups were, respectively, 0.124 \pm 0.071 , $-0.074 \pm 0.066$, and $0.072 \pm 0.052$. The profiles of correlations between \%BSC and current $\mathrm{T}$ differed from the null profile in all three groups (Low, $p<0.001$; Medium, $p<0.001$; High, $p<0.001$; FDR corrected; Fig. 3B, Extended Data Fig. 3-2). Results of repeated-measures ANOVA showed that the Fisher $z$ transformed correlation coefficient profiles (\%BSC vs current T) were different among the three groups for both contrasts (Angry: $F_{(2,48)}=7.9, p=0.001$; Ambiguous: $\left.F_{(2,48)}=63.6, p<0.001\right)$. Post hoc tests with Holm correction decomposing this profile difference revealed that the correlation coefficient profile of the Low group was higher than in the Medium and High groups in both contrast conditions (Angry: $p_{(\text {Low versus Medium })}=0.007, p_{(\text {Low versus High) }}=$ $0.007, p_{(\text {Medium versus High })}=0.967$; Ambiguous: $p_{(\text {Low versus Medium })}<$ $\left.0.001, p_{(\text {Low versus High })}=0.009, p_{(\text {Medium versus High })}<0.001\right)$.

\section{Pubertal T modulated the correlation between current $\mathrm{T}$ and \%BSC in the amygdala}

In the Angry-Control contrast, \%BSC values in both the left and right amygdala were positively correlated with current $\mathrm{T}$ in the Low group, but not in Medium or High groups (Left amygdala: $r_{(\text {Low })}=0.196, p_{(\text {Low })}=0.030 ; r_{(\text {Medium })}=-0.004$, $p_{(\text {Medium })}=0.964 ; r_{(\mathrm{High})}=0.000, p_{(\mathrm{High})}=0.998 ;$ Right $\mathrm{amyg}-$ dala: $r_{(\text {Low })}=0.235, p_{(\text {Low })}=0.009 ; r_{(\text {Medium })}=-0.027$, $\left.p_{(\text {Medium })}=0.762 ; r_{(\text {High })}=-0.041, p_{(\text {High })}=0.653\right)$. In the Ambiguous-Control contrast, \%BSC in the right amygdala was positively correlated with current $\mathrm{T}$ in the Low group, but not in the Medium or High group $\left(r_{(\text {Low })}=0.209, p_{(\text {Low })}\right.$ $=0.020 ; r_{(\text {Medium })}=-0.022, p_{(\text {Medium })}=0.807 ; r_{(\text {High })}=0.148$, $\left.p_{(\text {High })}=0.101\right)$; while in the left amygdala, positive correlation was found in the High group, but not the Low or Medium group $\left(r_{(\text {Low })}=0.129, p_{(\text {Low })}=0.156 ; r_{(\text {Medium })}=-\right.$ $\left.0.043, p_{(\text {Medium })}=0.631 ; r_{(\mathrm{High})}=0.185, p_{(\mathrm{High})}=0.041\right)$. Note that the apparent left-right differences in these relationships were not significant (Low group, $p=0.275$; High group, $p=0.585$ ).
Pubertal T modulated the correlation between current $\mathrm{T}$ and node strength across 25 ROIs

The profiles of interaction term (i.e., pubertal $\mathrm{T} \times$ current $\mathrm{T}$ ) were different from the null profile in both the Angry and Ambiguous face network (angry: $p<0.001$; ambiguous: $p<0.001)$. In the "angry-face network," the mean ( $\pm \mathrm{SD})$ partial correlation coefficients between current T and strength of the 25 ROIs in the Low, Medium, and High groups were, respectively, $0.040 \pm 0.059,-0.121 \pm 0.077$, and $-0.164 \pm 0.053$. The profiles of correlations between strength and current $\mathrm{T}$ differed from the null profile in all three groups (Low, $p=0.002$; Medium, $p<0.001$; High, $p<0.001$; FDR corrected; Fig. $4 A$, Extended Data Fig. 4-1). In the "ambiguous-face network," the mean partial correlation coefficients in the Low, Medium, and High groups were, respectively, $0.047 \pm 0.046,-0.106 \pm 0.088$, and $-0.086 \pm 0.075$. The profiles of correlations between strength and current $\mathrm{T}$ differed from the null profile in all three groups (Low, $p<0.001$; Medium, $p<0.001$; High, $p<0.001$; FDR corrected; Fig. 4B, Extended Data Fig. 4-1). Repeated-measures ANOVA results showed that the Fisher $z$-transformed correlation coefficient profiles (strength vs current $\mathrm{T}$ ) were different among the three pubertal-T groups (Angry: $F_{(2,48)}=65.3, p<0.001$; Ambiguous: $\left.F_{(2,48)}=27.7, p<0.001\right)$. Post hoc tests with Holm correction decomposed this profile difference and revealed that the correlation coefficient profile of the Low group was higher than those of the Medium and High groups in both face networks (Angry: $p_{\text {(Low versus Medium) }}<0.001, p_{\text {(Low versus High) }}<0.001$, $p_{(\text {Medium versus High })}=0.031$; Ambiguous: $p_{(\text {Low versus Medium })}<$ $\left.0.001, p_{(\text {Low versus High })}<0.001, p_{(\text {Medium versus High })}=0.450\right)$.

\section{Current $\mathrm{T}$ and brain response in the eye-related and eye- nonrelated sets of voxels}

In angry-face ROIs, $5.07 \%$ of voxels overlapped with an angryeye map, which showed regions related to participants viewing motion in the eye region in the observed angry faces. The overlapping voxels were located at anterior and posterior STS, FFA, LOC, V2V3, and amygdala. In ambiguous-face ROIs, only $0.79 \%$ of voxels overlapped with ambiguous-eye map, the overlapping voxels were mainly located in the cerebellum, MDLFC, and posterior STS. 

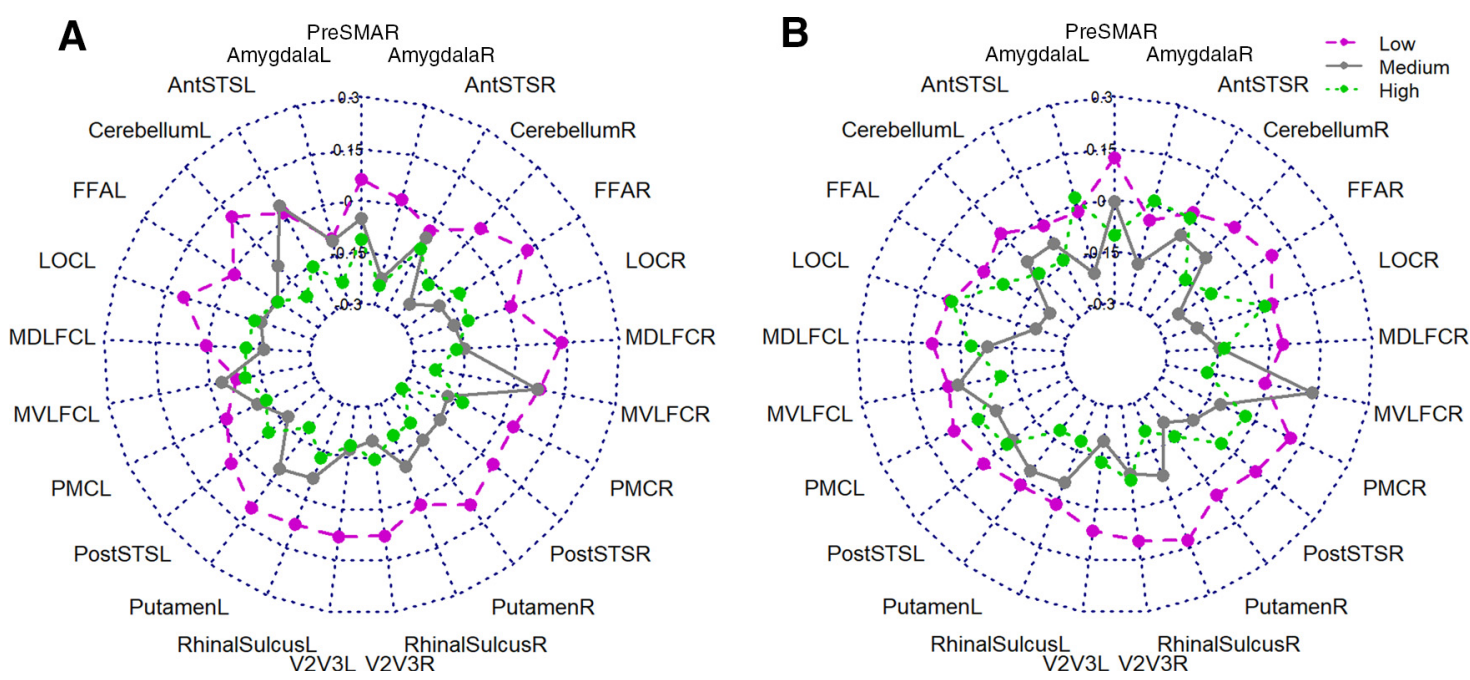

Figure 4. $\quad \boldsymbol{A}, \boldsymbol{B}$, Partial correlation coefficients between strength and current $\mathrm{T}$ for three groups: angry face $(\boldsymbol{A})$; ambiguous face $(\boldsymbol{B})$. Magenta dashed line represents Low group; gray solid line represents the Medium group; green dotted line represents the High group. Correlation coefficients for each region can be found in Extended Data Figure 4-1.

Table 1. Results of correlation between \%BSC and current $\mathrm{T}$ in eye-related and eye-nonrelated sets of voxels

\begin{tabular}{lllrrll}
\hline Contrast & Group & Voxel sets & \multicolumn{1}{l}{ Slope } & $R$ & $p$ & $p_{\text {adjusted }}$ (FDR) \\
\hline Angry vs Control & \multirow{2}{*}{ Low } & Eye-related & 6.485 & 0.234 & 0.009 & 0.027 \\
& & eye-nonrelated & 1.991 & 0.170 & 0.06 & 0.179 \\
& \multirow{4}{*}{ Medium } & Eye-related & -1.079 & -0.034 & 0.703 & 0.703 \\
& & eye-nonrelated & -0.038 & -0.003 & 0.972 & 0.972 \\
& \multirow{4}{*}{ High } & Eye-related & -1.419 & -0.052 & 0.56 & 0.703 \\
& & eye-nonrelated & -0.038 & -0.004 & 0.966 & 0.972 \\
Ambiguous vs & \multirow{2}{*}{ Low } & Eye-related & 5.142 & 0.170 & 0.059 & 0.088 \\
Control & & eye-nonrelated & 1.147 & 0.087 & 0.335 & 0.335 \\
& \multirow{2}{*}{ Medium } & Eye-related & -6.935 & -0.235 & 0.007 & 0.021 \\
& & eye-nonrelated & -3.117 & -0.248 & 0.005 & 0.014 \\
& \multirow{2}{*}{ High } & Eye-related & 1.665 & 0.063 & 0.485 & 0.485 \\
& & eye-nonrelated & 1.176 & 0.110 & 0.218 & 0.328 \\
\hline
\end{tabular}

For Angry versus Control contrast, the \%BSC in eye-related sets of voxels, which are related to participants' viewing motion in the eye region in the angry faces, positively correlated with current $\mathrm{T}$ in the Low group, but not in the other two groups; $\%$ BSC in eye-nonrelated sets of voxels did not correlate with current $\mathrm{T}$ in any groups (Table 1 ). The bootstrap test confirmed the difference on slopes of current $\mathrm{T}$ and \%BSC in eye-related versus eye-nonrelated sets of voxels $(p=0.010)$ in the Low Testosterone group (Fig. 5). There was no such pattern in the Ambiguous versus Control contrast in any of the three groups.

\section{Discussion}

According to the organizational-activational hypothesis, the organizational effects of testosterone on the developing brain moderate activational effects of testosterone on social behavior in adulthood. Our results showed that, in young men, the level of testosterone exposure during adolescence moderated the relationship between adult testosterone and brain response to faces in terms of the \% BSC and nodal strength of regions constituting the face network. Specifically, the relationship between current $\mathrm{T}$ and brain response to faces consistently tend to be positive only in participants who experienced low exposure to testosterone during their puberty.

With a large sample size, our data show in a robust manner that the relationship between adult levels of testosterone and face

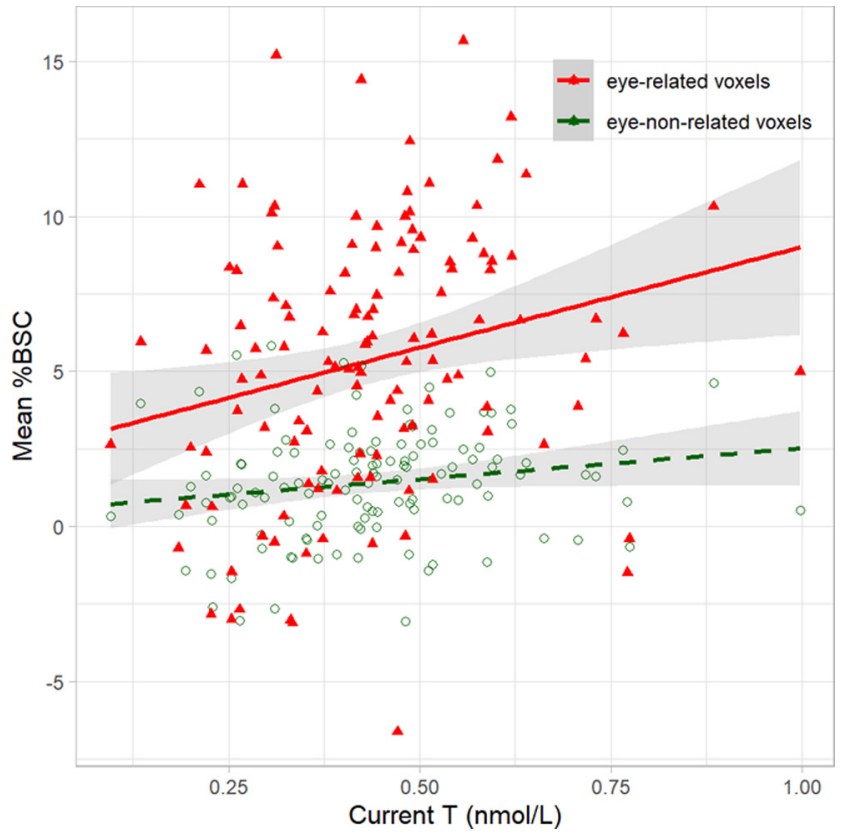

Figure 5. Correlation between \%BSC of Angry versus Control and current $\mathrm{T}$ in eye-related and eye-nonrelated sets of voxels in Low group.

processing is "primed" by pubertal testosterone. This moderating effect of pubertal testosterone is consistent with previous animal studies, which revealed that the sensitivity of social behaviors to adult testosterone is facilitated by testosterone exposure levels (and timing) in puberty (Sisk and Zehr, 2005; Schulz and Sisk, 2016). The direction of the moderation in these studies does not appear consistent, however, with that observed in our (human) study. Specifically, in the animal studies, castrated mice without testosterone treatment during puberty have a lower response to acute testosterone treatment in adulthood (mating and socially aggressive behavior) than those with testosterone treatment during puberty (Schulz and Sisk, 2006; Schulz et al., 2009b). In the present study, the (positive) relationship between current $\mathrm{T}$ and brain response to faces (i.e., higher current $\mathrm{T}$, greater brain response) was stronger in participants with low pubertal testosterone than those with high pubertal testosterone. Furthermore, 
the (negative) relationship between current $\mathrm{T}$ and functional connectivity (i.e., higher current $\mathrm{T}$, lower connectivity) was stronger in participants who had high pubertal testosterone. These differences between the findings of previous animal studies and this report may be related to differences in the behaviors under study: reproduction-related behaviors in animal studies, face processing here. Furthermore, the effects of testosterone on social behavior and cognition in human studies may depend on social contexts: in certain conditions it motivates rebellious, aggressive and violent behavior, while in other conditions it motivates prosocial behavior (Eisenegger et al., 2011). Even in the same social context, the effects of testosterone on social cognition may differ in men and women. For instance, using a similar "Reading the Mind in the Eyes" Test paradigm, some studies showed that acute administration of testosterone impaired empathy in women (Van Honk et al., 2011; Bos et al., 2016), while others reported that testosterone administration tended to increase empathy in men (Nadler et al., 2019).

Because of the lack of human studies on the organizational effects of pubertal testosterone and different task design in previous research, we can only speculate about possible interpretations of our findings. Previous findings have indicated that testosterone administration in healthy women promotes dominant gaze behavior when looking at angry faces (Terburg et al., 2012). Moreover, such dominance-enhancing effects of testosterone are particularly strong in individuals with socially submissive behavior. The administration of a single dose of testosterone reduced gaze avoidance in women with social anxiety disorder (Enter et al., 2016), a disorder associated with low levels of (endogenous) testosterone (Giltay et al., 2012). On the other hand, the same testosterone treatment tended to diminish fixations on the eyes in healthy women (Enter et al., 2016). Participants in the Low Puberty T group may display less social dominant behaviors (Rowe et al., 2004; Tarter et al., 2009; Crone and Dahl, 2012). Individuals with high current $\mathrm{T}$ in this group may tend to have prolonged eye contact with faces, which would lead to a higher brain response in those face-related regions, especially the amygdala and superior temporal sulcus, two regions known to respond to direct gaze (George et al., 2001; Nummenmaa and Calder, 2009).

Consistent with the above literature, we would expect-in individuals with low pubertal testosterone-a stronger correlation between current $\mathrm{T}$ and brain response to faces in a part of the face network that is associated with processing social cues carried by the eyes of an observed face. This is what we observed in the case of viewing angry (but not ambiguous) faces. Furthermore, the results with regard to the relationship between current $\mathrm{T}$ and the amygdala are also consistent with this explanation. The amygdala may play a role in the monitoring or perception of gaze contact; a higher fMRI response in this region was found in response to a directed, compared with an averted, gaze (Hooker et al., 2003; Burra et al., 2013). Our results showed that current $\mathrm{T}$ was positively associated with an amygdala response to angry faces in the Low Puberty-T group, indicating the possible prolonged eye contact during the task in participants with high adult testosterone in this group.

Prolonged eye contact may not only enhance the brain response to faces but also functional connectivity in the face network. Our analyses showed that current $\mathrm{T}$ tended to be negatively associated with functional connectivity in the Medium and High pubertal T group: the higher testosterone levels were associated with lower connectivity in these two groups. Reduction of face task-based functional connectivity was shown to correlate with lower direct eye contact in previous studies (Von Dem Hagen et al., 2014; Hadjikhani et al., 2017; Ziaei et al., 2017). The results also fit with prior research reporting that testosterone administration reduced face task-based functional connectivity in adults (van Wingen et al., 2010; Bos et al., 2016). Such a reduction is also consistent with testosterone-induced impairment in conscious recognition of facial expression (Van Honk and Schutter, 2007) and with the ability to infer mental states of another by looking into the eyes (Van Honk et al., 2011).

Though previous animal studies provide strong evidence of interaction between organizational and activational effects of testosterone, the underlying mechanism is unknown. The modulation of cell number and cell group volume are potential mechanisms for organizational effects of pubertal T. Compared with male hamsters with normal endogenous testosterone during puberty, hamsters who were not exposed to pubertal testosterone had a lower volume of the anterior ventral amygdala and the anterior dorsal medial amygdala, but a higher volume of the posterior dorsal medial amygdala (De Lorme et al., 2012). At a molecular level, pubertal $\mathrm{T}$ may alter the expression of genes including, but not limited to, the androgen receptor gene $(A R)$. Studies of cross-sex hormone therapy showed that the $A R$ expression in female patients with gender dysphoria decreased after testosterone treatment (Aranda et al., 2017). An epigenetic study also showed that hundreds of $\mathrm{CpG}$ sites, which were enriched in genomic loci containing androgen targets and in genes involved in tissue development, were more methylated in male than female participants (at three developmental periods: birth, and at 7 and 17 years of age), and indicated the possible contribution of androgen exposure to the regulation of gene expression (Suderman et al., 2017). This finding points to the complexity of the modulation effects of pubertal $\mathrm{T}$ on the relationship between adult testosterone and brain response to faces.

Our study has several limitations. The main one is the inclusion of males only. This is because of the focus on pubertal testosterone and, in turn, the focus of a grant funding this project. The other limitation is the lack of information about perinatal levels of testosterone and, therefore, possible variation in the perinatal organizational effects. The use of indirect proxies of prenatal testosterone exposure, such as the $2 \mathrm{D} / 4 \mathrm{D}$ ratio, are not without problems (Berenbaum et al., 2009; Richards et al., 2019). Access to direct testosterone measures (via amniocentesis) is possible only when required for clinical reasons (Bergman et al., 2010) and, as such, is not available in population-based studies.

Nonetheless, taking advantage of the relatively large sample, with testosterone assessed in a longitudinal manner throughout adolescence, our findings support the organizational-activational hypothesis by providing strong evidence of an interaction between pubertal testosterone and adult testosterone on face processing. It suggests that pubertal $\mathrm{T}$ may be an unknown modifier in studies investigating the activational effects of testosterone on social cognition in adulthood. Because of the paucity of human studies on pubertal testosterone, it is too early to make conclusions about how pubertal sex steroids moderate the sensitivity to sex hormones in adulthood. Future work about the differences on social cognition and behavior between individuals with low pubertal testosterone and those with high pubertal testosterone should be conducted. Similar longitudinal studies of female adolescents are also needed. Finally, molecular mechanisms may be explored using various transcriptomic and epigenetic approaches.

\section{References}

Adolphs R (2002) Neural systems for recognizing emotion. Curr Opin Neurobiol 12:169-177.

Aranda G, Fernández-Rebollo E, Pradas-Juni M, Hanzu FA, Kalko SG, Halperin I, Mora M (2017) Effects of sex steroids on the pattern of 
methylation and expression of the promoter region of estrogen and androgen receptors in people with gender dysphoria under cross-sex hormone treatment. J Steroid Biochem Mol Biol 172:20-28.

Berenbaum SA, Bryk KK, Nowak N, Quigley CA, Moffat S (2009) Fingers as a marker of prenatal androgen exposure. Endocrinology 150:5119-5124.

Bergman K, Glover V, Sarkar P, Abbott DH, O'Connor TG (2010) In utero cortisol and testosterone exposure and fear reactivity in infancy. Horm Behav 57:306-312.

Bos PA, Hofman D, Hermans EJ, Montoya ER, Baron-Cohen S, van Honk J (2016) Testosterone reduces functional connectivity during the "Reading the Mind in the Eyes" Test. Psychoneuroendocrinology 68:194-201.

Boyd A, Golding J, Macleod J, Lawlor DA, Fraser A, Henderson J, Molloy L, Ness A, Ring S, Smith GD (2013) Cohort profile: the "children of the 90s"-The index offspring of the Avon Longitudinal Study of Parents and Children. Int J Epidemiol 42:111-127.

Brambilla DJ, Matsumoto AM, Araujo AB, McKinlay JB (2009) The effect of diurnal variation on clinical measurement of serum testosterone and other sex hormone levels in men. J Clin Endocrinol Metab 94:907-913.

Buades-Rotger M, Engelke C, Beyer F, Keevil BG, Brabant G, Krämer UM (2016) Endogenous testosterone is associated with lower amygdala reactivity to angry faces and reduced aggressive behavior in healthy young women. Sci Rep 6:38538.

Burra N, Hervais-Adelman A, Kerzel D, Tamietto M, de Gelder B, Pegna AJ (2013) Amygdala activation for eye contact despite complete cortical blindness. J Neurosci 33:10483-10489.

Buzkova P (2016) Interaction testing: residuals-based permutations and parametric bootstrap in continuous, count, and binary data. Epidemiol Methods 5:119-128.

Crone EA, Dahl RE (2012) Understanding adolescence as a period of socialaffective engagement and goal flexibility. Nat Rev Neurosci 13:636-650.

Dalton KM, Nacewicz BM, Johnstone T, Schaefer HS, Gernsbacher MA, Goldsmith HH, Alexander AL, Davidson RJ (2005) Gaze fixation and the neural circuitry of face processing in autism. Nat Neurosci 8:519-526.

De Lorme KC, Schulz KM, Salas-Ramirez KY, Sisk CL (2012) Pubertal testosterone organizes regional volume and neuronal number within the medial amygdala of adult male Syrian hamsters. Brain Res 1460:33-40.

Derntl B, Windischberger C, Robinson S, Kryspin-Exner I, Gur RC, Moser E, Habel U (2009) Amygdala activity to fear and anger in healthy young males is associated with testosterone. Psychoneuroendocrinology 34:687693.

Eichmann F, Holst DV (1998) Organization of territorial marking behavior by testosterone during puberty in male tree shrews. Physiol Behav 65:785-791.

Eisenegger C, Haushofer J, Fehr E (2011) The role of testosterone in social interaction. Trends Cogn Sci 15:263-271.

Enter D, Terburg D, Harrewijn A, Spinhoven P, Roelofs K (2016) Single dose testosterone administration alleviates gaze avoidance in women with social anxiety disorder. Psychoneuroendocrinology 63:26-33.

Esteban O, Birman D, Schaer M, Koyejo OO, Poldrack RA, Gorgolewski KJ (2017) MRIQC: advancing the automatic prediction of image quality in MRI from unseen sites. PLoS One 12:e0184661.

Esteban O, Markiewicz CJ, Blair RW, Moodie CA, Isik AI, Erramuzpe A, Kent JD, Goncalves M, DuPre E, Snyder M, Oya H, Ghosh SS, Wright J, Durnez J, Poldrack RA, Gorgolewski KJ (2019) fMRIPrep: a robust preprocessing pipeline for functional MRI. Nat Methods 16:111-116.

Fraser A, Macdonald-wallis C, Tilling K, Boyd A, Golding J, Davey smith G, Henderson J, Macleod J, Molloy L, Ness A, Ring S, Nelson SM, Lawlor DA (2013) Cohort profile: the Avon longitudinal study of parents and children: ALSPAC mothers cohort. Int J Epidemiol 42:97-110.

Gamer M, Büchel C (2009) Amygdala activation predicts gaze toward fearful eyes. J Neurosci 29:9123-9126.

George N, Driver J, Dolan RJ (2001) Seen gaze-direction modulates fusiform activity and its coupling with other brain areas during face processing. Neuroimage 13:1102-1112.

Giltay EJ, Enter D, Zitman FG, Penninx BWJH, van Pelt J, Spinhoven P, Roelofs K (2012) Salivary testosterone: associations with depression, anxiety disorders, and antidepressant use in a large cohort study. J Psychosom Res 72:205-213.

Grosbras MH, Paus T (2006) Brain networks involved in viewing angry hands or faces. Cereb Cortex 16:1087-1096.

Guyer AE, Silk JS, Nelson EE (2016) The neurobiology of the emotional adolescent: from the inside out. Neurosci Biobehav Rev 70:74-85.
Hadjikhani N, Zurcher NR, Lassalle A, Hippolyte L, Ward N, Johnels JÅ (2017) The effect of constraining eye-contact during dynamic emotional face perception-an fMRI study. Soc Cogn Affect Neurosci 12:1197-1207.

Haxby JV, Hoffman EA, Gobbini MI (2000) The distributed human neural system for face perception. Trends Cogn Sci 4:223-233.

Herting MM, Gautam P, Spielberg JM, Kan E, Dahl RE, Sowell ER (2014) The role of testosterone and estradiol in brain volume changes across adolescence: a longitudinal structural MRI study. Hum Brain Mapp 35:5633-5645.

Hietanen JK (2018) Affective eye contact: an integrative review. Front Psychol 9:1587.

Holm S (1979) A simple sequentially rejective multiple test procedure. Scand J Stat 6:65-70.

Hooker CI, Paller KA, Gitelman DR, Parrish TB, Mesulam MM, Reber PJ (2003) Brain networks for analyzing eye gaze. Brain Res Cogn Brain Res 17:406-418.

Itier RJ, Batty M (2009) Neural bases of eye and gaze processing: the core of social cognition. Neurosci Biobehav Rev 33:843-863.

Jensen SKG, Pangelinan M, Björnholm L, Klasnja A, Leemans A, Drakesmith M, Evans CJ, Barker ED, Paus T (2018) Associations between prenatal, childhood, and adolescent stress and variations in white-matter properties in young men. Neuroimage 182:389-397.

Khairullah A, Klein LC, Ingle SM, May MT, Whetzel CA, Susman EJ, Paus T (2014) Testosterone trajectories and reference ranges in a large longitudinal sample of male adolescents. PLoS One 9:e108838.

Mandal MK, Awasthi A (2015) Understanding facial expressions in communication: cross-cultural and multidisciplinary perspectives. New Delhi: Springer India.

Manuck SB, Marsland AL, Flory JD, Gorka A, Ferrell RE, Hariri AR (2010) Salivary testosterone and a trinucleotide (CAG) length polymorphism in the androgen receptor gene predict amygdala reactivity in men. Psychoneuroendocrinology 35:94-104.

McHugh ML (2012) Interrater reliability: the kappa statistic. Biochem Med 22:276-282.

Nadler A, Camerer CF, Zava DT, Ortiz TL, Watson NV, Carré JM, Nave G (2019) Does testosterone impair men's cognitive empathy? Evidence from two large-scale randomized controlled trials. Proc Biol Sci 286:20191062

Nummenmaa L, Calder AJ (2009) Neural mechanisms of social attention. Trends Cogn Sci 13:135-143.

Pemberton JR (1975) Retention of mercurial preservatives in desiccated biological products. J Clin Microbiol 2:549-551.

Phoenix CH, Goy RW, Gerall AA, Young WC (1959) Organizing action of prenatally administered testosterone propionate on the tissues mediating mating behavior in the female guinea pig. Endocrinology 65:369-382.

Pruim RH, Mennes M, van Rooij D, Llera A, Buitelaar JK, Beckmann CF (2015) ICA-AROMA: A robust ICA-based strategy for removing motion artifacts from fMRI data. Neuroimage 112:267-277.

Richards G, Gomes M, Ventura T (2019) Testosterone measured from amniotic fluid and maternal plasma shows no significant association with directional asymmetry in newborn digit ratio (2D:4D). J Dev Orig Health Dis 10:362-367.

Rowe R, Maughan B, Worthman CM, Costello EJ, Angold A (2004) Testosterone, antisocial behavior, and social dominance in boys: pubertal development and biosocial interaction. Biol Psychiatry 55:546-552

Schulz KM, Sisk CL (2006) Pubertal hormones, the adolescent brain, and the maturation of social behaviors: lessons from the Syrian hamster. Mol Cell Endocrinol 254-255:120-126.

Schulz KM, Sisk CL (2016) The organizing actions of adolescent gonadal steroid hormones on brain and behavioral development. Neurosci Biobehav Rev 70:148-158.

Schulz KM, Richardson HN, Zehr JL, Osetek AJ, Menard TA, Sisk CL (2004) Gonadal hormones masculinize and defeminize reproductive behaviors during puberty in the male Syrian hamster. Horm Behav 45:242-249.

Schulz KM, Molenda-Figueira HA, Sisk CL (2009a) Back to the future: the organizational-activational hypothesis adapted to puberty and adolescence. Horm Behav 55:597-604.

Schulz KM, Zehr JL, Salas-Ramirez KY, Sisk CL (2009b) Testosterone programs adult social behavior before and during, but not after, adolescence. Endocrinology 150:3690-3698.

Schumann G, Loth E, Banaschewski T, Barbot A, Barker G, Büchel C, Conrod PJ, Dalley JW, Flor H, Gallinat J, Garavan H, Heinz A, Itterman 
B, Lathrop M, Mallik C, Mann K, Martinot J-L, Paus T, Poline J-B, Robbins TW, et al. (2010) The IMAGEN study: reinforcement-related behaviour in normal brain function and psychopathology. Mol Psychiatry 15:1128-1139.

Shrenker P, Maxson SC, Ginsburg BE (1985) The role of postnatal testosterone in the development of sexually dimorphic behaviors in DBA/1Bg mice. Physiol Behav 35:757-762.

Sisk CL, Zehr JL (2005) Pubertal hormones organize the adolescent brain and behavior. Front Neuroendocrinol 26:163-174.

Södergård R, Bäckström T, Shanbhag V, Carstensen H (1982) Calculation of free and bound fractions of testosterone and estradiol-17 beta to human plasma proteins at body temperature. J Steroid Biochem 16:801-810.

Spielberg JM, Forbes EE, Ladouceur CD, Worthman CM, Olino TM, Ryan ND, Dahl RE (2015) Pubertal testosterone influences threat-related amygdala-orbitofrontal cortex coupling. Soc Cogn Affect Neurosci 10:408-415.

Stanton SJ, Wirth MM, Waugh CE, Schultheiss OC (2009) Endogenous testosterone levels are associated with amygdala and ventromedial prefrontal cortex responses to anger faces in men but not women. Biol Psychol $81: 118-122$.

Suderman M, Simpkin A, Sharp G, Gaunt T, Lyttleton O, McArdle W, Ring S, Smith GD, Relton C (2017) Sex-associated autosomal DNA methylation differences are wide-spread and stable throughout childhood. bioRxiv 118265. doi: 10.1101/118265.

Tahmasebi AM, Artiges E, Banaschewski T, Barker GJ, Bruehl R, Büchel C, Conrod PJ, Flor H, Garavan H, Gallinat J, Heinz A, Ittermann B, Loth E, Mareckova K, Martinot J-L, Poline J-B, Rietschel M, Smolka MN, Ströhle A, Schumann G, et al. (2012) Creating probabilistic maps of the face network in the adolescent brain: a multicentre functional MRI study. Hum Brain Mapp 33:938-957.
Tarter RE, Kirisci L, Gavaler JS, Reynolds M, Kirillova G, Clark DB, Wu J, Moss HB, Vanyukov M (2009) Prospective study of the association between abandoned dwellings and testosterone level on the development of behaviors leading to cannabis use disorder in boys. Biol Psychiatry 65:116-121.

Terburg D, Aarts H, van Honk J (2012) Testosterone affects gaze aversion from angry faces outside of conscious awareness. Psychol Sci 23:459-463.

Van Honk J, Schutter DJLG (2007) Testosterone reduces conscious detection of signals serving social correction: implications for antisocial behavior. Psychol Sci 18:663-667.

Van Honk J, Schutter DJ, Bos PA, Kruijt AW, Lentjes EG, Baron-Cohen S (2011) Testosterone administration impairs cognitive empathy in women depending on second-to-fourth digit ratio. Proc Natl Acad Sci U S A 108:3448-3452.

van Wingen G, Mattern C, Verkes RJ, Buitelaar J, Fernández G (2010) Testosterone reduces amygdala-orbitofrontal cortex coupling. Psychoneuroendocrinology 35:105-113.

Vijayakumar N, Op de Macks Z, Shirtcliff EA, Pfeifer JH (2018) Puberty and the human brain: insights into adolescent development. Neurosci Biobehav Rev 92:417-436.

Vijayakumar N, Pfeifer JH, Flournoy JC, Hernandez LM, Dapretto M (2019) Affective reactivity during adolescence: associations with age, puberty and testosterone. Cortex 117:336-350.

Von Dem Hagen EAH, Stoyanova RS, Rowe JB, Baron-Cohen S, Calder AJ (2014) Direct gaze elicits atypical activation of the theory-of-mind network in Autism spectrum conditions. Cereb Cortex 24:1485-1492.

Wallen K (2009) The organizational hypothesis: reflections on the 50th anniversary of the publication of Phoenix, Goy, Gerall, and Young (1959). Horm Behav 55:561-565.

Ziaei M, Ebner NC, Burianová H (2017) Functional brain networks involved in gaze and emotional processing. Eur J Neurosci 45:312-320. 